\title{
SLAM-BASED BACKPACK LASER SCANNING FOR FOREST PLOT MAPPING
}

\author{
Jie Shao ${ }^{1,2}$, Wuming Zhang ${ }^{3,4, *}$, Lei Luo ${ }^{5}$, Shangshu Cai ${ }^{1}$, Hailang Jiang ${ }^{1}$ \\ ${ }^{1}$ State Key Laboratory of Remote Sensing Science, Beijing Engineering Research Center for Global Land Remote Sensing Products, \\ Institute of Remote Sensing Science and Engineering, Faculty of Geographical Science, Beijing Normal University, Beijing, China - \\ (shaojie, sscai, hljiang)@mail.bnu.edu.cn \\ 2 IRIT, CNRS, University of Toulouse, Toulouse, France \\ ${ }^{3}$ School of Geospatial Engineering and Science, Sun Yat-Sen University, Zhuhai, China - zhangwm25@mail.sysu.edu.cn \\ ${ }^{4}$ Southern Marine Science and Engineering Guangdong Laboratory (Zhuhai), Guangdong, China \\ ${ }^{5}$ Key Laboratory of Digital Earth Science, Aerospace Information Research Institute, Chinese Academy of Sciences, Beijing, China \\ - luolei@radi.ac.cn
}

\section{Commission II, WG II/4}

KEY WORDS: Forest mapping, LiDAR, Backpack laser scanning, Point cloud, SLAM

\begin{abstract}
:
Acquisition of three-dimensional (3D) structural information is significant for forest measurements. To achieve faster data collection in forests, we design a backpack laser scanning (BLS) system using a single mobile laser scanning (MLS) scanner and specific to forest environments. The simultaneous localization and mapping (SLAM) approach based on the natural geometric characteristics of trees is used for BLS-based forest mapping, in which the skeleton line of the individual tree is extracted for scan matching and the incremental maps are adopted for global optimization of all the BLS point clouds. The final experimental results show that the SLAM-based BLS system achieves accurate forest plots mapping and allows reaching low mapping errors, in which the mean errors are approximately 3 $\mathrm{cm}$ in the horizontal and $2 \mathrm{~cm}$ in the vertical direction.
\end{abstract}

\section{INTRODUCTION}

Three-dimensional (3D) structural information acquisition is an essential prerequisite and is the key to digital forest inventories (Chambers et al., 2007). Light detection and ranging (LiDAR) has been suggested to be a practical technique to acquire precise 3D structural information of the forest (Zolkos et al., 2013; Magnussen et al., 2015; Varvia et al., 2019; Luo et al, 2019). Mobile laser scanning (MLS) has gained attention in forest mapping because of the advantage of the immensely faster data collection (Liang et al., 2014). The main limitation of MLS appears during the mapping step.

Most of the existing MLS-based mapping techniques are based on the global navigation satellite system/inertial measurement unit (GNSS-IMU) techniques, in which the GNSS system is used for positioning the MLS system, and the IMU provides attitude information for the orientation of the MLS system (Tang et al., 2015). However, the mapping becomes difficult when the GNSS signal is weaken or blocked. Thus, the simultaneous localization and mapping (SLAM) technique is typically used for solving the mapping, which is the process of mapping an unknown environment and locating the mobile platform simultaneously (Dissanayake et al, 2001). Kukko et al. (2017) used SLAM technique to correct the GNSS-IMU trajectory for position drift and in turn, the initial trajectory obtained from the GNSS-IMU system provided the constraints for the SLAM. The method not only obtained forest mapping but also improved the positioning accuracy, whereas the GNSS signal loss caused by the occlusions may affect the performance. The feature-based SLAM methods commonly extract features in environments to achieve mapping but without the GNSS-IMU system. For instance, the LOAM method (Zhang and Singh, 2014) extracted the line and plane features from object surfaces for positioning and mapping in indoor and urban scenarios. However, due to the complexity of forest environments and the similarity between the objects, the reliable features are difficult to extract from the tree surface, and inaccurate corresponding pairs would fall scan matching into a local optimum. In addition, some methods registered forest point clouds, including the methods based on artificial markers (Henning and Radtke, 2006; Hilker et al., 2012; Zhang et al., 2016a) and automated marker-free methods (Kelbe et al., 2016; Liu et al., 2017). These related methods were typically proposed for the registration of dense terrestrial laser scanning (TLS) point clouds and solved the occlusion influencing from single-scan TLS data (Wan et al., 2019), which are ill-suited for matching of sparse MLS point clouds.

To achieve MLS-based forest mapping, we design a backpack laser scanning (BLS) system using a single MLS scanner and propose a novel SLAM method based on the natural geometric characteristics of trees. We first integrate a simple BLS system without GNSS-IMU system for acquisition of forest point clouds. Then, the trunk skeleton lines that represent the tree center lines are extracted for matching BLS point clouds, which prevents inaccurate correspondence. At last the graph-based optimization is used for the elimination of accumulative errors.

\section{METHODS}

In this paper, we realize forest plot mapping using the designed BLS system, in which the key of this task is to solve the SLAM problem. The SLAM process involves feature extraction, scan matching, and optimization. For scan matching, we propose the combination of the line features and point features for estimating the transformation between adjacent BLS point clouds, in which

\footnotetext{
* Corresponding author
} 
the line features are derived from trunk skeletons that are natural geometric elements of trees, and the point features are extracted from each BLS point cloud evenly. Moreover, the incremental BLS point clouds and trunk skeleton nodes are used to provide constraints for optimization of all the BLS point clouds. The process of the paper is shown in Figure 1.

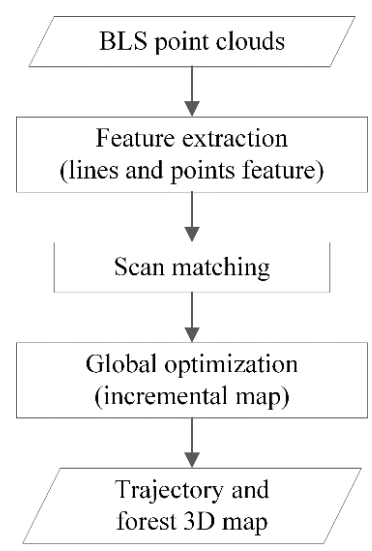

Figure 1. The flowchart

\subsection{Feature extraction}

Feature selection and feature extraction are the precondition of feature-based SLAM method. To achieve accurate matching of BLS point clouds in forests, we propose to combine the trunk skeleton line and point features, in which the line and point features are used for maintaining the horizontal and vertical matching accuracies, respectively.

For line features, we utilize a hierarchical clustering method to extract the trunk skeleton lines. First, the BLS scan is divided into multiple subsets based on the vertical angular resolution. Then, region growing is used to cluster the adjacent points in each subset, and the circle fit based on the least square method is used to detect trunk skeleton nodes, in which the connected line between two neighbor nodes is regarded as a line feature (Figure 2a). In addition, the difference of Gaussian (DoG) is used to extract point features. In this paper, we referred to the work of Theiler et al. (2014) for extracting point features (Figure 2b).

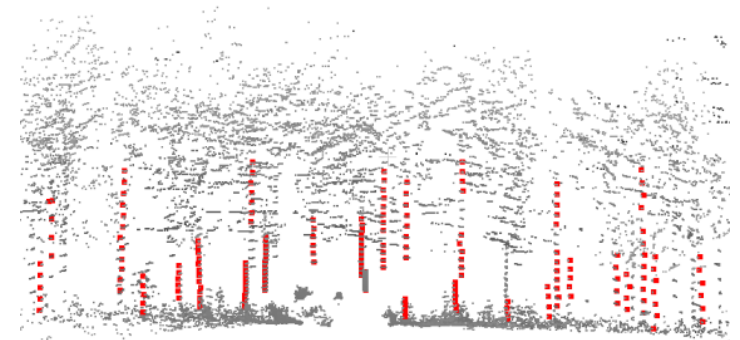

(a)

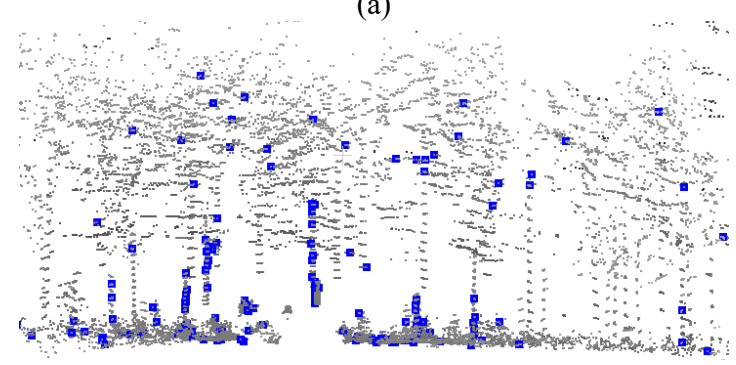

(b)

Figure 2. Feature extraction (grey points are the BLS points). (a) represents the skeleton nodes (red points), (b) represents the even-distributed point features (blue points).

\subsection{Scan matching}

The process of scan matching is to construct correspondence and calculate transformation between the adjacent BLS point clouds. Because the acquisition of BLS data is continuous, we use the Euclidean nearest neighbor search to find corresponding feature pairs, in which the distance between the corresponding pair is regarded as the constraint. For line features, the point-to-line (skeleton node to skeleton line) correspondence is established, and the point-to-line distance $d_{l}$ can be computed by

$$
d_{l}=\frac{\left|\left(X_{(t+1, l)}-X_{(t, a)}\right) \times\left(X_{(t+1, l)}-X_{(t, b)}\right)\right|}{\left|X_{(t, a)}-X_{(t, b)}\right|}
$$

where $\quad X_{(t+1, l)}$ is a skeleton node at the time of sweep $t+1$ $X_{(t, a)}$ and $X_{(t, b)}$ are the two nearest skeleton points of $X_{(t+1, l)}$ at the time of sweep $t$

Because of the fast error convergence speed, the point-to-plane correspondence is established (Low, 2004), in which the plane is consist of three nearest points of the extracted point feature that are within a certain neighborhood and not on the same line. Therefore, the point-to-plane distance $d_{p}$ can be computed by

$$
d_{p}=\frac{\left|\left(X_{(t+1, p)}-X_{(t, a)}\right) \cdot\left(\left(X_{(t+1, p)}-X_{(t, b)}\right) \times\left(X_{(t+1, p)}-X_{(t, c)}\right)\right)\right|}{\left|\left(X_{(t+1, p)}-X_{(t, b)}\right) \times\left(X_{(t+1, p)}-X_{(t, c)}\right)\right|}
$$

where $\quad X_{(t+1, p)}$ is a point feature at the time of sweep $t+1$, and its corresponding plane at the time of sweep $t$, is set to $\left\{X_{(t, a)}, X_{(t, b)}, X_{(t, c)}\right\}$

$\overrightarrow{\boldsymbol{n}}$ is the normal vector of the plane

Let $X_{t}$ be the extracted feature at the time of sweep $t$, and $X_{t+1}$ be the correspondence at the time of sweep $t+1$. A rigid transformation relationship between $X_{t}$ and $X_{t+1}$ can be established:

$$
X_{t+1}=\boldsymbol{R} X_{t}+\boldsymbol{T}
$$

where

$\boldsymbol{R}$ and $\boldsymbol{T}$ are unknown and can be represented by 6 degrees of freedom (6-DOF)

$\boldsymbol{R}$ is the rotation matrix $\left(\boldsymbol{R} \in \mathbb{R}^{3 \times 3}\right)$

$\boldsymbol{T}$ is the translation vector

Then, the unknown can be solved by minimizing the error $e$ toward zero with the Levenberg-Marquardt (L-M) method:

$$
e=\arg \min _{e} \frac{1}{2} \sum_{i=1}^{N}\left\|d_{i}-0\right\|^{2}
$$

Final, we can calculate the unknown through nonlinear iterations.

\subsection{Scan optimization}

To mitigate the accumulative error, we propose an optimization framework based on two incremental maps. The two maps contain incremental trunk skeleton points and incremental BLS point clouds. In particular, the first frame of BLS point cloud and its corresponding trunk skeleton points are regarded as reference, 
and its coordinate system is also regarded as the common coordinate system. For each of the subsequent BLS point clouds, all of the previous BLS point clouds and trunk skeleton points in the common coordinate system are respectively composed of two incremental maps and regarded as constraints for optimizing the current frame of BLS point cloud. Meanwhile, the optimized BLS point cloud and its corresponding trunk skeleton points are added to the previous data, and two new incremental maps can be built. Furthermore, all BLS point clouds can be transformed in the common coordinate system.

\section{RESULTS AND DISCUSSION}

\subsection{Data acquisition}

In this study, we designed a BLS system that consists of a single MLS scanner (Velodyne VLP-16), and a simple backpack frame (see Figure 3 ). The VLP-16 collects 600,000 points/sec, and its field of view is $360^{\circ}$ in the horizontal direction and $30^{\circ}$ in the vertical direction. The maximum range is approximately $100 \mathrm{~m}$. We acquired two sets of data in two plots (Plot A and Plot B) of $30 \mathrm{~m} \times 30 \mathrm{~m}$ in size.

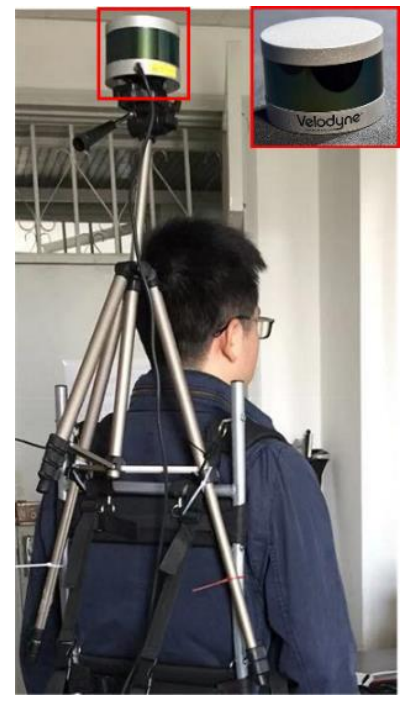

Figure 3. BLS system

\subsection{Scan matching results}

According to the proposed method, we first matched the adjacent BLS scans and calculated the transformation between them. We arbitrarily selected two adjacent scans as instances and obtained the transformation matrix, in which we described the matrix $\boldsymbol{M}$ by $6-\mathrm{DOF}$,

$$
\boldsymbol{M}=[-0.013,0.050,-0.446,0.087,-0.030,-0.002]
$$

where $\boldsymbol{M}(1) 、 \boldsymbol{M}(2)$ and $\boldsymbol{M}(3)$ represent rotation angles around the $x$-, $y$-, and $z$-axes.

$\boldsymbol{M}(4) 、 \boldsymbol{M}(5)$ and $\boldsymbol{M}(6)$ are translations along the $x$-, $y$-, and $z$-axes.

Then, the two adjacent scans can be matched based on the matrix Figure 4 shows the matching results, in which Figure 4(a) shows the initial states of the two adjacent scans and Figure $4(b)$ is the matching result. As seen in the results, the proposed method can achieve accurate scan matching.

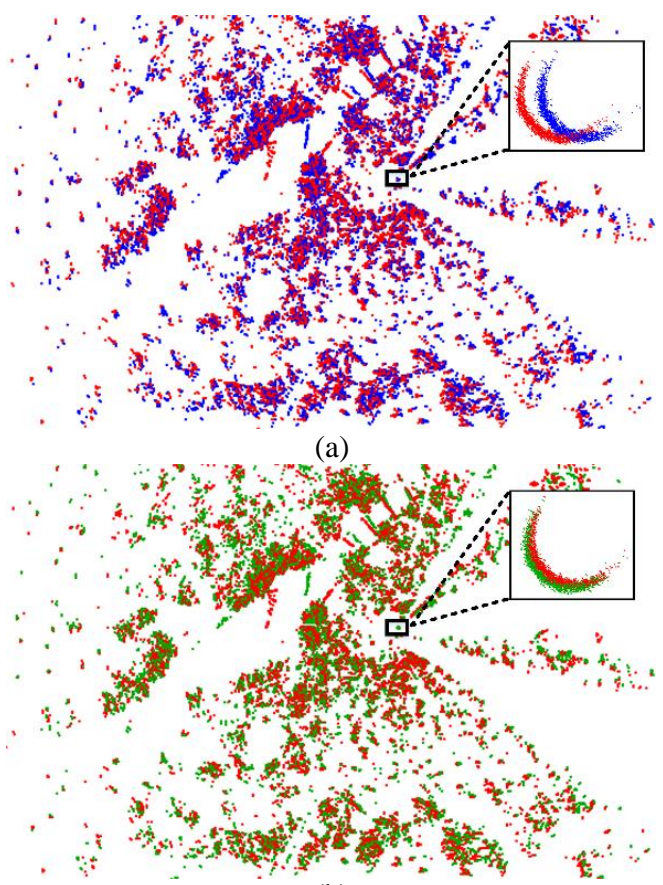

(b)

Figure 4. Scan matching results (red points are references, blue points in (a) and green points in (b) are the matched points).

\subsection{Forest plot mapping}

To verify the effectiveness of the proposed method, we achieved forest plots mapping and recovered the trajectory of the BLS system based on the acquired data (Figure 5).

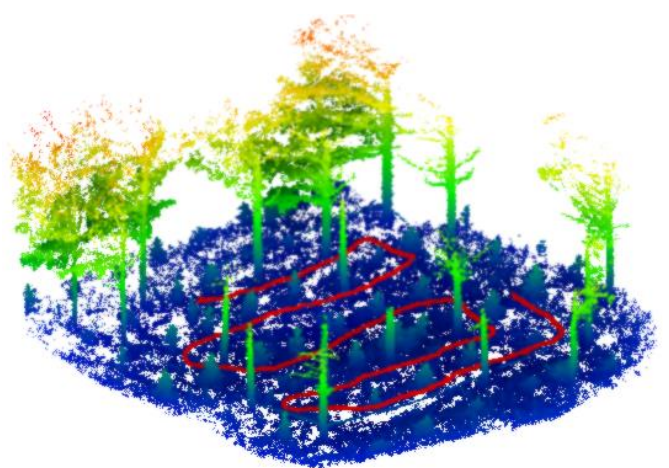

(a)

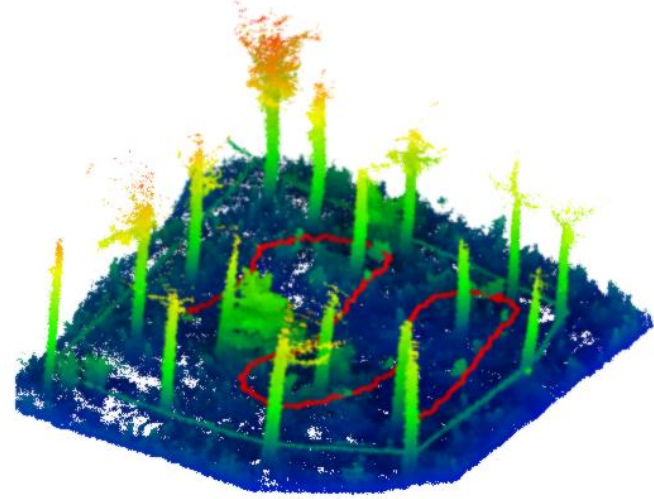

(b)

Figure 5. Forest mapping results (grey points are forest points and red curves are the trajectories). (a) is the mapping result of Plot A, and (b) is the result of Plot B. 
From Figure 5, the results show that the below-canopy structures are mapped by using the proposed method, and the trajectories are coincident with the practical movements. In the mapping results, each tree in the plots is registered accurately, and the distribution of individual trees has no significant deviations, which suggest that the trees reconstructed by the proposed method are available. In addition, the open trajectories of the scanner do not drift from their correct values and are recovered by the proposed method in the two plots, which suggests the reliability of the trajectories.

\subsection{Mapping accuracy}

In forests, the locations of trees were available for evaluating horizontal accuracy. To quantitatively evaluate the performance of the proposed method, the tree position from the BLS-based mapping results were compared to those from the corresponding multi-scan TLS data. Therefore, each BLS-based mapping result was manually registered with its corresponding multi-scan TLS data. Then, the tree position is represented by extracting the center of the cross section of point cloud that falls above the ground level. In practice, we first filtered the ground points and the non-ground points (Zhang et al., 2016b), and detected all individual trees. In addition, the position deviations, including mean deviation (Mean), root mean square error (RMSE), and the maximum deviation (Max), were used to assess horizontal accuracy. The results are summarized in Table 1.

\begin{tabular}{ccccc}
\hline Plots & $\begin{array}{c}\text { Number of } \\
\text { sample trees }\end{array}$ & \multicolumn{3}{c}{ Deviations (m) } \\
\cline { 3 - 5 } & 10 & Mean & RMSE & Max \\
\hline $\mathrm{A}$ & 10 & 0.029 & 0.031 & 0.040 \\
$\mathrm{~B}$ & 0.025 & 0.027 & 0.036 \\
\hline
\end{tabular}

Table 1. Horizontal accuracy

From Table 1, the deviations were at the centimeter level, of which the mean absolute deviations and RMSE values were approximately $0.03 \mathrm{~m}$, and the maximum deviations were less than $0.04 \mathrm{~m}$, which indicated highly accurate mapping results. In addition, to further assess the performance of the proposed method, the deviations between the branch position in the BLSbased mapping results and the corresponding positions in the multi-scan TLS data were calculated for evaluating the vertical accuracy. In practice, some sample points on the branches were selected to calculate the deviations. The vertical accuracies are summarized in Table 2.

\begin{tabular}{ccccc}
\hline Plots & Number of & \multicolumn{3}{c}{ Deviations (m) } \\
\cline { 3 - 5 } & sample points & Mean & RMSE & Max \\
\hline A & 10 & 0.0179 & 0.0187 & 0.0283 \\
B & 10 & 0.0153 & 0.0155 & 0.0189 \\
\hline
\end{tabular}

Table 2. Vertical accuracy

In the test plots, the branch position deviations were also at the centimeter level, of which the mean absolute deviations and RMSE values varied between $0.015 \mathrm{~m}$ and $0.020 \mathrm{~m}$. In general, the requirement of the vertical accuracy is lower than the horizontal accuracy for forest measurements (e.g., tree height), so the accuracy results indicated the effectiveness of the results. Overall, the proposed optimization framework can achieve BLSbased forest mapping, of which the incremental maps provide effective global consistency constraints for each frame of BLS point cloud. In particular, the trunk skeletons map maintained the accuracies of positioning and mapping in the horizontal direction, and the incremental BLS point clouds that are in common coordinate system provided effective constraint for forest mapping in the vertical direction.

\section{DISCUSSION}

To demonstrate the advantage in scan matching, we compare the matching results from the proposed method with those from the iterative closest point (ICP) algorithm (Besl and Mckav, 1992). Specifically, the locations of tree stems were used for evaluating the horizontal accuracy (Table 3), and ground and vegetation points were selected for evaluating the vertical accuracy of the matching results (Table 4).

\begin{tabular}{cccccc}
\hline Plots & Methods & Trees & \multicolumn{3}{c}{ Stem distance $(\mathrm{m})$} \\
\cline { 4 - 6 } & & number & mean & RMSE & Max \\
\hline A & ICP & 10 & 0.041 & 0.043 & 0.059 \\
& Ours & 10 & 0.022 & 0.023 & 0.041 \\
B & ICP & 10 & 0.051 & 0.053 & 0.069 \\
& Ours & 10 & 0.018 & 0.019 & 0.027 \\
\hline
\end{tabular}

Table 3. Horizontal accuracy

\begin{tabular}{cccccc}
\hline Plots & Methods & Points & \multicolumn{3}{c}{ Distance (m) } \\
\cline { 4 - 6 } & & number & mean & RMSE & Max \\
\hline A & ICP & 10 & 0.062 & 0.076 & 0.128 \\
& Ours & 10 & 0.007 & 0.009 & 0.015 \\
B & ICP & 10 & 0.057 & 0.062 & 0.112 \\
& Ours & 10 & 0.008 & 0.010 & 0.018 \\
\hline
\end{tabular}

Table 4. Vertical accuracy

The above results show that the mean distances and RMSEs of the proposed method are approximately $0.02 \mathrm{~m}$ in the horizontal direction and are approximately $0.01 \mathrm{~m}$ in the vertical direction. In the results, the matching accuracies of the proposed method are significantly higher than those of the ICP method, in which the proposed method adopted the trunk skeleton lines to provide horizontal constraint and obtained high horizontal accuracy, and the even-distributed points maintained the matching accuracy in the vertical direction. The ICP method utilized the overlapping points to calculate transformation between two adjacent scans, but the sparsity of the BLS point cloud caused the inaccurate correspondences and scan matching. The results indicated the effectiveness of the proposed method in scan matching.

\section{CONCLUSIONS}

To achieve fast forest mapping, this paper designed a single scanner BLS system and proposed a new mapping method. The BLS system is simple and specific to forest environments. In addition, the line features derived from the trunk skeletons was proposed and used for matching of the BLS point clouds, and the line feature effectively solved the inaccurate registration problem caused by inaccurate matching pairs in the horizontal direction; meanwhile, the optimization framework based on the incremental BLS point clouds and the tree trunk skeleton lines provided effective constraints for the BLS-based SLAM in forest environments and ensured the positioning accuracies without a GNSS-IMU system or loop-closure detection process, of which the incremental skeleton lines maintained accurate registration of individual trees, especially tree stems. Subsequently, practical experimental results show the effectiveness and reliability of the designed BLS system and the proposed method. Although the method achieved fast data acquisition, the data acquired by a single scanner were limited below the canopy. To acquire more complete data, two scanners will be considered, of which one is used for the SLAM technique and below-canopy data acquisition and another is used for canopy data acquisition. 


\section{ACKNOWLEDGEMENTS}

This work was supported by the National Natural Science Foundation of China, grant nos. 41671414 and 41971380 . This work was also supported by Guangxi Natural Science Fund for Innovation Research Team (grant no.2019JJF50001) and the Open Fund of State Key Laboratory of Remote Sensing Science (grant no.OFSLRSS201920).

\section{REFERENCES}

Besl, P.J., Mckay, N.D., 1992. A method for registration of 3-D shapes. IEEE T. Pattern Anal. 14(2), 239-256.

Chambers, J.Q., Asner, G.P., Morton, D.C., et al. 2007. Regional ecosystem structure and function: ecological insights from remote sensing of tropical forests. Trends in Ecology \& Evolution 22, 414-423.

Dissanayake, M.W.M.G., Newman, P., Clark, S., Durrant-Whyte, H.F., Csorba, M., 2001. A solution to the simultaneous localization and map building (SLAM) problem. IEEE Transactions on Robotics and Automation 17(3), 229-241.

Henning, J.G., Radtke, P.J., 2006. Detailed stem measurements of standing trees from ground-based scanning lidar. Forest Sci. 52(1), 67-80.

Hilker, T., Coops, N.C., Culvenor, D.S., Newnham, G., Wulder, M.A., Bater, C.W., Siggins, A., 2012. A simple technique for coregistration of terrestrial lidar observations for forestry applications. Remote Sens. Lett. 3(3), 239-247.

Liang, X., Hyyppä, J., Kukko, A., Kaartinen, H., Jaakkola, A., Yu, X., 2014. The use of a mobile laser scanning system for mapping large forest plots. IEEE Geosci. Remote Sens. Lett. 11, 1504-1508.

Liu, J., Liang, X., Hyyppä, J., Yu, X., Lehtomäki, M., Pyörälä, J., Zhu, L., Wang, Y., Chen, R., 2017. Automated matching of multiple terrestrial laser scans for stem mapping without the use of artificial references. Int. J. Appl. Earth Obs. Geoinf. 56, 1323.

Low, K., 2004. Linear least-squares optimization for point-toplane ICP surface registration. Chapel Hill, University of North Carolina 4(10).

Luo, L., Wang, X., Guo, H., Lasaponara, R., Zong, X., Masini, N., Wang, G., Shi, P., Khatteli, H., Chen, F., Tariq, S., Shao, J., Bachagha, N., Yang, R., Yao, Y., 2019. Airborne and spaceborne remote sensing for archaeological and cultural heritage application: a review of the century (1907-2017). Remote Sens. Environ. 232, 111280.
Kelbe, D., Van Aardt, J., Romanczyk, P., Leeuwen, M.V., CawseNicholson, K., 2016. Marker-free registration of forest terrestrial laser scanner data pairs with embedded confidence metrics. IEEE Trans. Geosci. Remote Sens. 54, 4314-4330.

Kukko, A., Kaijaluoto, R., Kaartinen, H., Lehtola, V.V., Jaakkola, A., Hyyppä, J., 2017. Graph SLAM correction for single scanner MLS forest data under boreal forest canopy. ISPRS J. Photogramm. Remote Sens. 132, 199-209.

Magnussen, S., Næsset, E., Gobakken, T., 2015. LiDAR supported estimation of change in forest biomass with time invariant regression model. Can. J. Forest Res. 45(11), 1514.

Shao, J., Zhang, W., Mellado, N., Wang, N., Jin, S., Cai, S., Luo, L., Lejemble, T., Yan, G., 2020. SLAM-based forest plot mapping combining terrestrial and mobile laser scanning. ISPRS J. Photogramm. Remote Sens. 163, 214-230.

Tang, J., Chen, Y., Niu, X., Wang, L., Chen, L., Liu, J., Shi, C., Hyyppä, J., 2015. LiDAR scan matching aided inertial navigation system in gnss-denied environments. Sensors 15, 16710-16728.

Theiler, P.W., Wegner, J.D., Schindler, K., 2014. Keypointbased 4-points congruent sets - automated marker-less registration of laser scans. ISPRS J. Photogramm. Remote Sens. 96, 149-163.

Varvia, P., Lahivaara, T., Maltamo, M., Packalen, P., Seppanen, A., 2019. Gaussian process regression for forest attribute estimation from airborne laser scanning data. IEEE Trans. Geosci. Remote Sens. 57 (6), 3361-3369.

Wan, P., Wang, T., Zhang, W., Liang, X., Skidmore, A.K., Yan, G., 2019. Quantification of occlusions influencing the tree stem curve retrieving from single-scan terrestrial laser scanning data. Forest Ecosystems. 6(1).

Zhang, J., Singh, S., 2014. LOAM: lidar odometry and mapping in real-time. Robotics: Science and Systems Conference.

Zhang, W., Chen, Y., Wang, H., Chen, M., Wang, X., Yan, G., 2016a. Efficient registration of terrestrial lidar scans using a coarse-to-fine strategy for forestry applications. Agric. For. Meteorol. 225, 8-23.

Zhang, W., Qi, J., Wan, P., Wang, H., Xie, D., Wang, X., Yan, G., 2016b. An easy-to-use airborne LiDAR data filtering method based on cloth simulation. Remote Sens. 8, 501.

Zolkos, S.G., Goetz, S.J., Dubayah, R., 2013. A meta-analysis of terrestrial aboveground biomass estimation using lidar remote sensing. Remote Sens. Environ. 128 (21), 289-298. 